\title{
Rate code input produces temporal code output from cockroach antennal lobes
}

\author{
William C. Lemon, Wayne M. Getz * \\ Division of Insect Biology, University of California, Berkeley, CA 94720-3112, USA
}

\begin{abstract}
The experiments presented here were designed to determine the origin of the temporally complex activity of antennal lobe projection neurons in the cockroach olfactory system. We determined this through the use of complex chemical stimuli that evoked neural activity recorded extracellularly from olfactory sensory neurons and intracellularly from antennal lobe projection neurons in the cockroach Periplaneta americana. Olfactory information was represented by a simple, short time-scale rate code in the olfactory sensory neurons. This rate code input from the sensory neurons was processed by the antennal lobe and transformed into a longer time-scale, temporally encoded output expressed across a smaller population of antennal lobe projection neurons. The projection neuron responses comprised temporal patterns of increases and decreases in spike frequency that differed among projection neurons and were consistent among repeated presentations of the same stimulus. Presentation of simple and complex chemical stimuli showed that the complexity of projection neuron activity was a product of the antennal lobes and was not associated with the chemical complexity of the stimulus. To characterize the encoding schemes used by each class of neurons, the responses were decomposed into their principal components. The stimulus was correlated with only the first principal component of the activity of sensory neurons, which is consistent with a rate encoding scheme. The stimulus was correlated with higher order principal components of the activity of projection neurons, which is consistent with a temporal encoding scheme. (c) 2000 Elsevier Science Ireland Ltd. All rights reserved.
\end{abstract}

Keywords: Insect olfaction; Neural coding; Projection neuron; Olfactory sensory neuron; Periplaneta americana

\section{Introduction}

Recent theoretic and experimental investigations indicate that spike trains may carry sensory information encoded as a temporal code or a rate code (Rieke et al., 1997; Softky, 1995; Theunissen and Miller, 1995). A temporal code, which em-

\footnotetext{
* Corresponding author. Tel.: + 1-510-6428745; fax: + 1510-6427428.

E-mail address: getz@nature.berkeley.edu (W.M. Getz).
}

ploys information encoded in the timing of the spikes, can carry information at a higher density than a rate code, which carries information in the average rate of spikes. This suggests that a single neuron employing a temporal code could transmit an amount of information that would occupy many neurons if it were carried by a rate code. At points of convergence or divergence in the nervous system, therefore, information may be translated from one form of neural encoding to another. 
In insect olfactory systems (Fig. 1), the primary sensory neurons are the olfactory sensory neurons (OSNs) in the antennae. The axons of the OSNs project to the antennal lobe, which is analogous to the vertebrate olfactory bulb, where they synapse with a population of several hundred local interneurons (LNs) that then synapse with about 120 projection neurons (PNs) (Boeckh et al., 1990). The projection neurons carry olfactory information to the higher processing areas of the brain in the mushroom bodies and the lateral protocerebrum. In the antennal lobe, therefore, the olfactory information carried in a large population of OSNs must be translated into a form that can be represented in a relatively small number of PNs.

When cockroach antennae are presented with a chemical stimulus, each responsive $\mathrm{PN}$ produces a temporally complex phasic response that outlasts the stimulus (Laurent and Davidowitz, 1994; Laurent et al., 1996; Lemon and Getz, 1998). The temporal complexity of PN activity could have a number of sources. The PNs may simply follow OSN activity that represents the temporal qualities of the odor stimulus. Alternatively, synaptic interactions within the olfactory glomeruli (Boeckh et al., 1990; Christensen et al., 1996) may result in chemically simple stimuli evoking simple PN activity and chemically complex stimuli producing complex PN activity. Finally, the neural architecture of olfactory glomeruli could translate simple OSN input into complex PN output (Getz and Lutz, 1999). If translation from simple input

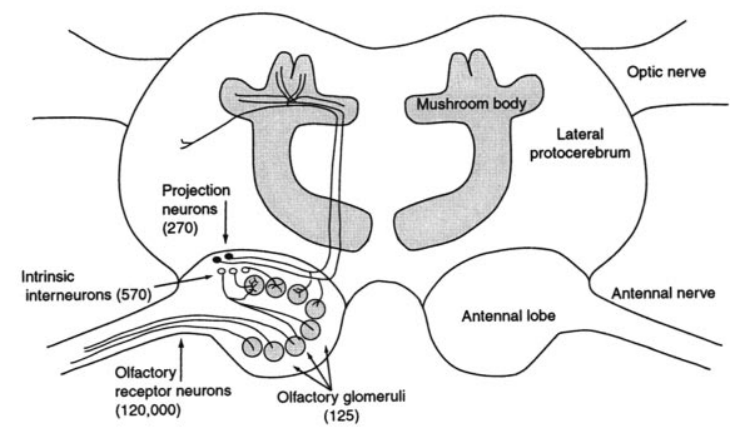

Fig. 1. Cartoon of the most notable structures in the cockroach olfactory system. to complex output is occurring, then the complexity seen in PN activity may be a dynamic temporal code representing a simple stimulus (Bialek and Rieke, 1992; Theunissen and Miller, 1995; Rieke et al., 1997). Presentation of simple and complex olfactory stimuli to cockroaches while recording from OSNs and PNs will establish whether the complexity seen in the neural activity of PNs is a product of the antennal lobe or a result of the chemical complexity of the stimulus.

The OSN and PN responses in this study were evaluated against the criteria established for qualification as rate codes and temporal codes (Theunissen and Miller, 1995). In brief, the criterion for rate encoding is that the encoded information must be correlated only with the average number of spikes within the encoding window (the smallest time period of neural activity in which the stimulus can be represented). The criterion for temporal coding is that the encoded information must be correlated with some aspect of the temporal pattern of spikes within the encoding window. In practice, neural responses are decomposed into their principal components. Typically, the first principal component is correlated with the spike rate while the second and higher order principal components represent aspects of the temporal patterning of the spike train (Theunissen and Miller, 1995). We show this correlation to hold for the data reported here. Thus, correlation of the stimulus with only the first principal component is usually indicative of a rate encoding scheme. Correlation of the stimulus with higher order principal components indicates a temporal encoding scheme.

\section{Methods}

\subsection{Stimulation}

Our stimulation techniques were similar to those previously used on honeybees and cockroaches (Getz and Akers, 1996; Lemon and Getz, 1997). Stimuli were delivered by passing a 60 $\mathrm{ml} / \mathrm{min}$ airstream through a cartridge containing odorant evaporating from $1 \mu \mathrm{g}$ of the liquid chemical. A clean air stream flowing over the 
target sensillum at $120 \mathrm{ml} / \mathrm{min}$ prevented stimulation between pulses of odor. In the present study each stimulus train consisted of two $200 \mathrm{~ms}$ pulses separated by $200 \mathrm{~ms}$ of clean air. Stimulus trains were presented at 1-2 min intervals. Each neuron was stimulated 10 times, five times with 1-hexanol and five times with coconut.

\subsection{OSN recordings}

Sensory neuron recordings were made from basiconic sensilla of the female cockroach, Periplaneta americana. We used extracellular recording techniques that have been described in detail (Lemon and Getz, 1997) to record the activity of single OSNs. Tungsten extracellular recording electrodes, electrolytically sharpened to less than 1 $\mu \mathrm{m}$ at the tip, were placed at the base of a sensillum. Signals were amplified differentially and digitized at $20 \mathrm{kHz}$ and stored directly on a microcomputer.

\section{3. $P N$ recordings}

Intracellular recordings were made from somata of antennal lobe PNs. The animals were cold anaesthetized and immobilized dorsal side up in a Sylgard lined dish divided into two separate wells. A window of cuticle was opened between the compound eyes leaving the antennae intact. The tracheae on the anterior face of the brain were removed and the sheath surrounding the antennal lobes was removed with fine forceps. One antenna was passed through a small opening to the adjoining well and the opening in the divider was sealed with petroleum jelly. The well containing the body of the cockroach was superfused with physiological saline at room temperature. The adjacent well, containing the distal portion of the antenna, remained dry allowing direct olfactory stimulation. Intracellular recordings were made with glass microelectrodes filled with $3 \mathrm{M}$ K-acetate. Final electrode resistance was 70-100 M $\Omega$. The antennal lobe was immobilized on a small stainless steel platform held beneath the brain with a micromanipulator. This technique allowed stable recordings from PN cell bodies, which action potentials passively invaded from the active regions of the neuron, for more than $1 \mathrm{~h}$. The activity of PNs was distinguished from the activity of LNs by the presence of large amplitude sodium spikes. In the cockroach antennal lobe, most LNs are non-spiking and transmit information by graded calcium 'spikelets' (Distler et al., 1998). During a recording session, the cell types were easily discriminated by the amplitudes of the depolarizing events. The PN activity was digitized at $20 \mathrm{kHz}$ and recorded directly on a microcomputer.

\subsection{Analysis}

For preliminary comparison of OSN and PN activity, responses were divided into $50 \mathrm{~ms}$ time bins and the total numbers of spikes that occurred during each bin were counted. The average spike frequency of each neuron in response to presentation of a stimulus of clean air was subtracted from each response to odor. These adjusted responses from the 5 repeated presentations of each odor stimulus were averaged for each neuron. The responses were normalized within each neuron and are presented here as the mean spike rate during each $50 \mathrm{~ms}$ bin. All analyses were performed with Matlab.

The responses of PNs and OSNs were analyzed to determine if they met the criteria for qualification as temporally encoded information. Stimulus reconstruction, in the traditional sense (Marmarelis and Marmarelis, 1978; Rieke et al., 1997), was not feasible because the stimulus waveform carried little temporal information. The lack of temporal dynamics makes it difficult to establish two parameters of the response upon which the criteria for temporal encoding depend: the integration window and the encoding window (Theunissen and Miller, 1995). For static olfactory stimuli that evoke responses that long outlast the stimulus, the duration of the integration and encoding windows are impossible to determine experimentally unless one already knows how odors are represented by the nervous system. We coarsely estimated that the integration and encoding windows were are on the order of $200 \mathrm{~ms}$ in duration. Analyzing the olfactory responses using windows of 100 and $400 \mathrm{~ms}$ did not alter the results presented here. 
The olfactory coding problem is primarily concerned with determining how the chemical identity of the stimulus is encoded, which is a parameter of the stimulus that is independent of the temporal structure of the stimulus. To determine how chemical identity is encoded by OSNs and PNs, we followed the procedure described by Theunissen and Miller (1995). In brief, individual spike trains were quantified and decomposed into a set of orthogonal waveforms known as principal components (PCs) that represent different levels of temporal complexity (Richmond and Optican, 1987a,b). The distributions of these PCs were then statistically compared to determine if they differed with the complexity of the stimulus odor.

Repeated stimulation of each neuron allowed us to obtain five waveforms (spike trains) representing responses to 1 -hexanol and 5 spike trains representing responses to coconut. The portion of each response analyzed began at stimulus onset and lasted $1000 \mathrm{~ms}$. These ten spike trains were then used to obtain the first two PCs. These two PCs represent orthogonal waveforms, the first of which (PC1) best represents the features common to the original ten waveforms and the second of which (PC2) best represents the features common to the residual after PC1 has been subtracted from each of the ten waveforms. Each waveform is characterized by 20 time bins of $50 \mathrm{~ms}$ duration and thus represents a point in 20 dimensional space. The PCs themselves represent orthogonal vectors in this 20 dimensional space and span a two dimensional subspace that accounts for more of the variation of the 10 points (10 original waveforms) than any other two dimensional subspace. The projection of any individual waveform in the 20 dimensional space onto this two dimensional space produces two coefficients, one associated with each of the PCs. In this way, we associate two PC coefficients with each waveform.

Next we implemented the following bootstrap procedure (Efron, 1982; Richmond and Optican, 1987a,b, 1990). From the group of 10 waveforms we drew five with replacement at random (this can be done in $5^{10} \approx 9.8$ million ways). We then generated the first two PCs associated with these 5 waveforms and the corresponding two PC projection coefficients associated with each of these 5 waveforms. We repeated this sampling and generation of PCs and associated coefficients 1000 times. Since each repeat generated $5 \mathrm{PC} 1$ and 5 PC2 coefficients, we finally obtained a distribution of 5000 PC1 and 5000 PC2 coefficients. We then sampled five waveforms with replacement from the five 1-hexanol waveforms alone (this can be done in $5^{5}=3125$ ways) and repeated this 1000 times. As in the previous case, this allowed us to generate a second distribution of $5000 \mathrm{PC} 1$ and 5000 PC2 coefficients. Finally we repeated the procedure with the five coconut waveforms to obtain a third distribution of 5000 PC1 and 5000 PC2 coefficients. These techniques provided a reasonable estimate of the distribution of PC coefficients (Efron, 1982).

Significant differences between the coefficients derived from the random stimuli and the coefficients derived from individual chemical stimuli indicated that the temporal modulation of individual responses were significantly dependent upon the chemical identity of the stimulus. If the first and second distributions of PC1 coefficients described above are significantly different, then we can conclude that waveforms generated by 1-hexanol produce values in PC1 coefficients that are significantly different from random. Similarly, significant differences between the first and third distributions of $\mathrm{PC} 1$ coefficients indicate that waveforms produced in response to coconut are significantly different from random.

Similar conclusions can be drawn from significant differences in the PC2 coefficients. Information theory predicts that sensory systems producing responses that have only a significant PC1, which is typically correlated with the spike rate, employ a rate encoding scheme, while systems producing responses that have significant higher order PCs employ a temporal encoding scheme (Theunissen and Miller, 1995). The distributions of PC coefficients were compared using the Komolgorov-Smirnoff test (Sokal and Rohlf, 1995). Recalling that these bootstrap procedures were applied to data from individual neurons, the procedure allowed us to test whether significantly different patterns of odor coding occur in each of the 20 OSNs and 18 PNs. 


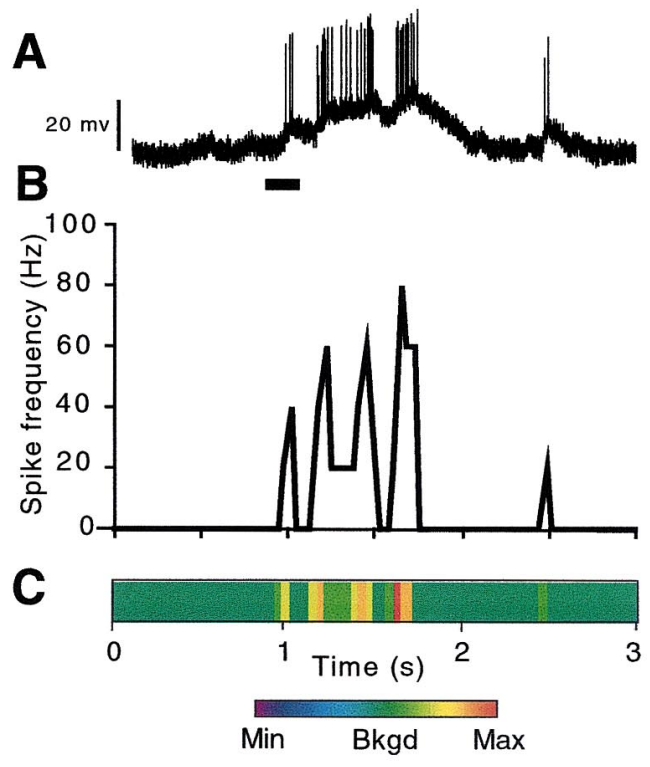

Fig. 2
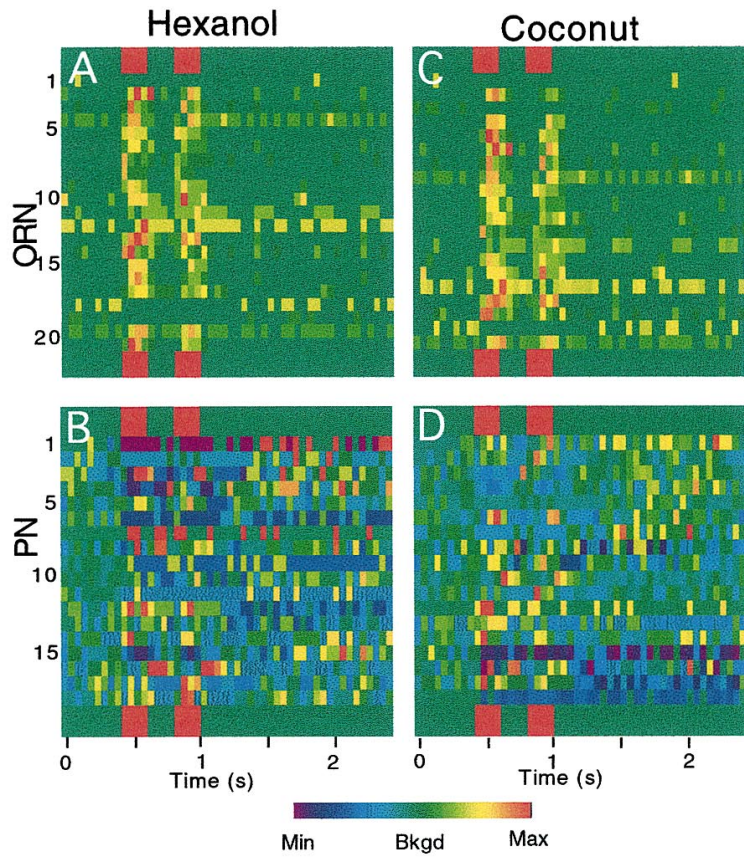

Fig. 3

Fig. 2. (Continued)

\section{Results}

\subsection{Olfactory responses}

Olfactory stimuli evoked $\mathrm{PN}$ responses that were complex and outlasted the stimulus (Fig. 2A). Spike frequencies varied with time, ranging from inactivity to rates approaching $100 \mathrm{~Hz}$ (Fig. 2B). To visualize the activity of a large population of neurons, the spike frequencies of each neuron were color coded and displayed in a linear array of $50 \mathrm{~ms}$ epochs (Fig. 2C).

The responses elicited from OSNs and PNs by the simple odorant 1-hexanol are illustrated using color codes (Fig. 3A and B). A population of OSNs responded with an increase in spike frequency that lasted only as long as the simple odor was present (Fig. 3A). A small proportion of OSNs had a low level of background activity that decreased with stimulus presentation. In contrast, the responses evoked by the same simple stimulus were much more variable in PNs (Fig. 3B). Some PNs increased their spike frequency with stimulus onset while others decreased their spike frequency. These response patterns were consistent within 4-5 repeated presentations to each neuron and varied among neurons. The variability of response properties was compared for repeated

Fig. 2. Examples of a response of a single neuron to an odorant and the subsequent analyses of that response. (A) Intracellular recording from the soma of a $\mathrm{PN}$ in response to a $200 \mathrm{~ms}$ pulse of 1-hexanol (bar below spike train) showing compound synaptic potentials and temporally patterned bursts of action potentials. (B) Spike frequencies obtained by averaging spike rates in each $50 \mathrm{~ms}$ time bin used to discretize the spike train. (C) Color-coded normalized spike frequencies for the spike train. Green represents the background-firing rate of that neuron in the absence of any stimulus. Warm colors represent increased spike frequency and cool colors represent decreased spike frequency.

Fig. 3. Normalized spike frequency for populations of OSNs and PNs responding to two $200 \mathrm{~ms}$ pulses (red squares at top and bottom) of 1-hexanol and coconut. Each horizontal series of colored boxes represents the firing rate of a single neuron averaged across 4-5 identical stimuli. Color code is the same as Fig. 2C. (A) Responses to 1-hexanol of the 20 OSNs that responded to 1-hexanol and coconut. (B) Responses to 1-hexanol of the 18 PNs that responded to 1-hexanol and coconut. (C) Responses to coconut of the same 20 OSNs shown in A. (D) Responses to coconut of the same 18 PNs shown in B. 
stimulus presentations within and among PNs by calculating the differences in spike frequency at every $50 \mathrm{~ms}$ bin for all possible pairs of evoked responses, both within a neuron and between different neurons. Analysis of variance (Sokal and Rohlf, 1995) indicated that the amount of variation in responses within a neuron was significantly less than the amount of variation among neurons $(F=149.1, \mathrm{df}=1480, \quad P<0.0001)$. In approximately $60 \%$ of the cockroach PNs that were studied, the spike frequency did not change in response to 1-hexanol. Those responses are not shown here.

The responses of OSNs and PNs elicited by the complex blend of odorants present in coconut (Fig. $3 \mathrm{C}$ and D) are equally as variable as the responses to 1-hexanol (Fig. 3A and B). Activity patterns evoked by coconut were consistent within 4-5 repeated presentations to each neuron with greater variation among different neurons. Like the responses to the simple odor, the complex odor blend of coconut evoked a brief increase in spike frequency in a population of the OSNs (Fig. 3C) but a more varied and complex response in PNs (Fig. 3D). In approximately $35 \%$ of the PNs, the spike frequency did not change in response to coconut.

\subsection{Rate coding vs. temporal coding}

The criteria for determining the encoding scheme of a sensory system as being either a rate encoding scheme or a temporal encoding scheme have been rigorously defined (Theunissen and Miller, 1995). The initial step in determining the encoding scheme of the cockroach antennal lobes was decomposition of the spike trains into PCs. The first two PCs were calculated for each OSN and PN and examples from two of each class of neuron are shown in Fig. 4. The coefficient of PC1 was highly correlated with the spike count of each response, while the PC2 coefficient was not correlated with spike count (Fig. 5A).

The coefficients of the PCs were compared to determine if they differed with the chemical complexity of the stimulus. The responses of PNs were grossly different (Fig. 3) and this comparison distinguished difference in spike rate from differences in spike train temporal dynamics. Examples of the distributions of coefficients of one OSN and

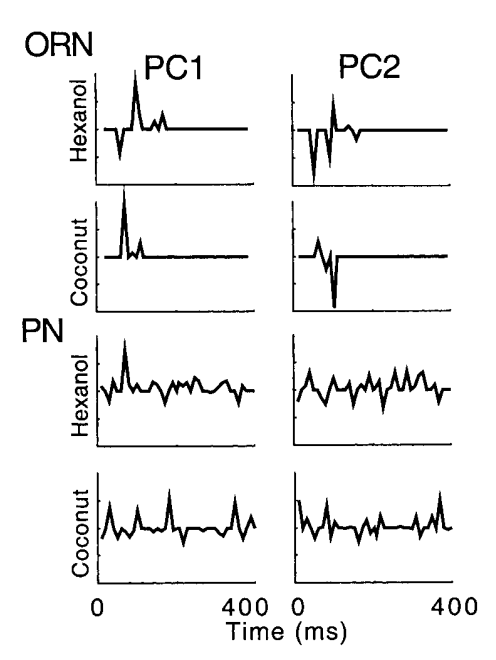

Fig. 4. Examples of the first two PCs derived from the responses of one OSN and one PN to stimulation with hexanol and coconut. The midline of each PC is at zero deviation from the average. The vertical scale is the same for both PCs.

one PN responding to hexanol, coconut and the pooled set of stimuli are shown in Fig. 5B.

The coefficients were dependent on the chemical identity of the stimulus odor, and OSNs and PNs differed in the effects that the stimulus quality had on the PC1 and PC2 coefficients (Table 1). Only a few OSNs responded differently to the two odors by altering the $\mathrm{PC} 1$ coefficients of their responses, or spike rates. This indicates that the firing rate of most OSNs carry only information about the presence of an appropriate odor and little information about the chemical identity of that odor. The latter information is only carried by the ensemble response of thousands of OSNs. The PC2 coefficients of OSN responses were not significantly different when stimulated with hexanol or coconut. The activity of OSNs simply indicates that an odorant to which the OSN is sensitive is present at the periphery. The PNs, on the other hand, responded to the two odors by changing the PC1 coefficients or the $\mathrm{PC} 2$ coefficients of their responses, or both. In addition, four PNs differed in their PC2 coefficients and not in their PC1 coefficients. The significant differences between the PC2 coefficients in response to hexanol and coconut indicate that PNs use a temporal encoding scheme to represent the chemical identity of the stimulus. 


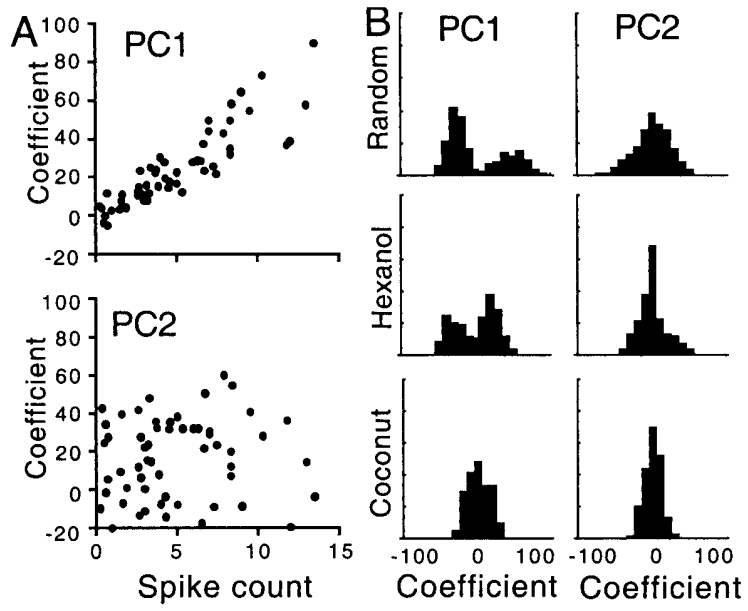

Fig. 5. (A) Correlation of mean spike count of OSN's and PN's with the first two PCs. PC1 is highly correlated with the mean spike count $(r=0.884, P<0.001)$. PC2 is not correlated with the mean spike count $(r=0.073, P>0.5)$. (B) Distributions of bootstrap PC coefficients of a single PN. The distributions of pooled coefficients were derived from a sample chosen randomly from the responses of the $\mathrm{PN}$ with no regard to the stimulus odor. The hexanol and coconut coefficients were derived from a similar samples of the PN's responses to the individual odors. The illustrated hexanol and coconut PC coefficients are significantly different from the random PC coefficients (Komolgorov-Smirnoff, $P<0.05$ for $\mathrm{PC} 1$ and PC2 comparisons). Not all neurons showed this dependence (Table 1).

\section{Discussion}

The stimulus reconstruction methods used in the analysis of many sensory systems do not address the fundamental problem of olfactory coding. These well developed methodologies obtain a best estimate of the transformation of the temporally dynamic stimulus waveform into the temporally dynamic neural response waveform.
The problem of olfactory coding requires that the temporal dynamics of the stimulus be integrated over some interval producing a descriptive, stable spike train that is independent of the temporal structure of the stimulus. The identities of a virtually unlimited number of combinations of odorants that an animal may encounter must be encoded in a relatively small number of PNs. This convergence of information requires that this small number of neurons carry more information than can be easily represented by a rate code. The results presented in this paper suggest that the responses of PNs are temporally complex spike trains that are not correlated with the temporal structure of the stimulus. This result is expected if the important information encoded by PNs is the chemical composition of the stimulus and not the temporal dynamics of the stimulus.

Several aspects of the antennal lobe PN code make stimulus reconstruction and analysis of temporal coding difficult. The duration of the encoding window is unknown. Evidence presented here and elsewhere indicates that PN activity long outlasts the duration of the stimulus, suggesting that the encoding window can be quite long. Nor can the duration of the encoding window be derived from the stimulus-response transformation (Marmarelis and Marmarelis, 1978; Theunissen and Miller, 1995; Rieke et al., 1997), as the stimulus has neither a relevant time or frequency component. Likewise, the duration of the integration time window remains unknown, but is probably shorter than the typical stimulus duration encountered in a natural odor plume and longer than the minimum stimulus duration that can be transduced by the OSNs (Lemon and Getz 1997).

Table 1

Dependence of the coefficients of PCs on the chemical identity of the stimulus

\begin{tabular}{|c|c|c|c|}
\hline Cell Type & Number & Number of significant PC1 coefficients ${ }^{\mathrm{a}}$ & Number of significant PC2 coefficients ${ }^{b}$ \\
\hline OSN & 20 & 3 & 0 \\
\hline $\mathrm{PN}$ & 18 & 10 & $12^{\mathrm{c}}$ \\
\hline
\end{tabular}

\footnotetext{
a Significantly different PC1 coefficients indicate that the spike rate was dependent on the stimulus.

b Significant PC2 coefficients indicate that the temporal pattern of the response was dependent on the stimulus.

${ }^{\mathrm{c}}$ Four PNs had significant PC2 coefficients without having significant PC1 coefficients.
} 
Analyses of the PCs of neural responses to olfactory stimuli indicate that OSNs employ a rate encoding scheme and PNs employ a temporal encoding scheme. The sensory neurons had significant PC1s and non-significant PC2s, which is diagnostic of a rate encoding sensory system (Theunissen and Miller, 1995). The encoding mechanism used by PNs, however, is more complex. Significant PC2s show that the stimulus is correlated with temporal dynamics of the PN response, which is a temporal encoding scheme. The temporal encoding scheme demonstrated by these neurons does not exclude the presence of temporal coding of the temporal dynamics of the stimulus (Theunissen and Miller, 1995). The activity of some PNs may carry information about the temporal structure of the stimulus and studies are under way to identify any such neurons and examine how that information is encoded.

In summary, both a chemically simple stimulus and a complex blend of odorants produce a simple rate code in the OSNs, the primary sensory afferents, that faithfully represent the temporal characteristics of the stimulus. The olfactory glomeruli integrate the short time-scale population rate codes of OSNs and translate them into longer time-scale temporal codes expressed across a smaller population of PNs. The temporal complexity seen in PN activity, therefore, is a dynamic temporal code produced by the synaptic interactions within the antennal lobe and does not represent complexity of the stimulus.

\section{Acknowledgements}

We thank Noel Holmgren, Rebecca Johnston, Gilles Laurent, Brian Smith and Frederic Theunissen for their helpful comments. This study was supported by NSF and DARPA.

\section{References}

Bialek, W., Rieke, F., 1992. Reliability and information transmission in spiking neurons. Trends Neurosci. 15, 428-434.

Boeckh, J., Distler, P., Ernst, K.D., Hösl, M., Malun, D., 1990. Olfactory bulb and antennal lobe. In: Schild, D.
(Ed.), Chemosensory Information Processing. SpringerVerlag, Berlin, pp. 201-227.

Christensen, T.A., Heinbockel, T., Hildebrand, J.G., 1996. Olfactory information processing in the brain: Encoding chemical and temporal features of odors. J. Neurobiol. 30, 82-91.

Distler, P.G., Gruber, C., Boeckh, J., 1998. Synaptic connections between GABA-immunoreactive neurons and uniglomerular projection neurons within the antennal lobe of the cockroach, Periplaneta americana. Synapse 29, 1-13.

Efron B., 1982, The jackknife, the bootstrap and other resampling plans (Society for Industrial and Applied Mathematics, Philadelphia).

Getz, W.M., Akers, R.P., 1996. Coding properties of peak and average response rates in American cockroach olfactory sensory cells. Biosystems 40, 55-63.

Getz, W.M., Lutz, A., 1999. A neural network model of general olfactory coding in the insect antennal lobe. Chem. Senses 24, 351-372.

Laurent, G., Davidowitz, H., 1994. Encoding of olfactory information with oscillating neural assemblies. Science 265, $1872-1875$.

Laurent, G., Wehr, M., Davidowitz, H., 1996. Temporal representations of odors in an olfactory network. J. Neurosci. $16,3837-3847$.

Lemon, W.C., Getz, W.M., 1997. Temporal resolution of general odor pulses by olfactory sensory neurons in American cockroaches. J. Exp. Biol. 200, 1809-1819.

Lemon, W.C., Getz, W.M., 1998. Responses of cockroach antennal lobe projection neurons to pulsatile olfactory stimuli. Ann. N.Y. Acad. Sci. 855, 517-520.

Marmarelis, P.Z., Marmarelis, V.Z., 1978. Analysis of physiological systems: The white-noise approach. Plenum Press, New York, p. 487.

Richmond, B.J., Optican, L.M., 1987a. Temporal encoding of two-dimensional patterns by single units in primate inferior temporal cortex. II. Quantification of response waveform. J. Neurophysiol. 57, 147-161.

Richmond, B.J., Optican, L.M., 1987b. Temporal encoding of two-dimensional patterns by single units in primate inferior temporal cortex. III. Information theoretic analysis. J. Neurophysiol. 57, 162-178.

Richmond, B.J., Optican, L.M., 1990. Temporal encoding of two-dimensional patterns by single units in primate primary visual cortex. II: Information transmission. J. Neurophysiol. 64, 370-380.

Rieke, F., Warland, D., de Ruyter van Steveninck, R., Bialek, W., 1997. Spikes: Exploring the neural code. MIT Press, Cambridge, Massachusetts, p. 395.

Softky, W.R., 1995. Simple codes versus efficient codes. Curr. Opin. Neurobiol. 5, 239-247.

Sokal, R.R., Rohlf, F.J., 1995. Biometry. W.H. Freeman, New York, p. 887.

Theunissen, F.E., Miller, J.P., 1995. Temporal encoding in nervous systems-a rigorous definition. J. Computat. Neurosci. 2, 149-162. 